\title{
Proof of the Landau-Zener Formula in an Adiabatic Limit with Small Eigenvalue Gaps
}

\section{George A. Hagedorn`}

Department of Mathematics, and Center for Transport Theory and Mathematical Physics, Virginia Polytechnic Institute and State University, Blacksburg, Virginia 24061-0123, USA

Received February 3, 1990; in revised form July 25, 1990

Abstract. We consider a smooth operator-valued function $H(t, \delta)$ that has two isolated non-degenerate eigenvalues $E_{\mathscr{A}}(t, \delta)$ and $E_{\mathscr{B}}(t, \delta)$ for $\delta>0$. We assume these eigenvalues are bounded away from the rest of the spectrum of $H(t, \delta)$, but have an avoided crossing with one another with a closest approach that is $O(\delta)$ as $\delta$ tends to zero. Under these circumstances, we study the small $\varepsilon$ limit for the adiabatic Schrödinger equation

$$
i \varepsilon \frac{\partial \psi}{\partial t}=H\left(t, \varepsilon^{1 / 2}\right) \psi
$$

We prove that the Landau-Zener formula correctly describes the coupling between the adiabatic states associated with the eigenvalues $E_{\mathscr{A}}(t, \delta)$ and $E_{\mathscr{B}}(t, \delta)$ as the system propagates through the avoided crossing.

\section{Introduction}

Adiabatic approximations in quantum mechanics describe solutions to Schrödinger equations with slowly varying time-dependent Hamiltonians. More precisely, if the time scale is chosen to be commensurate with the Hamiltonian's variation, then adiabatic approximations describe the small $\varepsilon$ behavior of solutions to the Schrödinger equation

$$
i \varepsilon \frac{\partial \psi}{\partial t}=H(t) \psi
$$

for $t$ in some fixed interval. The classical adiabatic theorem states that if $H(t)$ is a smooth family of self-adjoint operators with a continuous, isolated, multiplicity

\footnotetext{
* Supported in part by the National Foundation under Grant number DMS-8801360
} 
one eigenvalue $E(t)$, then the Schrödinger equation has a solution of the form

$$
\exp \left\{-i \int^{t} E(r) d r / \varepsilon\right\} \Phi(t)+O(\varepsilon),
$$

where $\Phi(t)$ is a particular smooth choice for the normalized eigenvector associated with $E(t)$. Under general circumstances, this result can be extended $[1,4,6,9,10,12]$ to an asymptotic expansion of the form

$$
\exp \left\{-i \int^{t} E(r) d r / \varepsilon\right\}\left(\Phi(t)+\varepsilon \psi_{1}(t)+\varepsilon^{2} \psi_{2}(t)+\cdots\right) \text {. }
$$

The Landau-Zener formula describes infinite order corrections to this expansion that are associated with small gaps between $E(t)$ and the rest of the spectrum of $H(t)$. In particular, suppose the gap between $E(t)$ and the rest of the spectrum of $H(t)$ attains its absolute minimum width $\delta$ at a unique point $t_{0}$ in the interval $-T \leqq t \leqq T$. Furthermore, assume $-T<t_{0}<T$, and that the width of the gap behaves like $\sqrt{\delta^{2}+a^{2}\left(t-t_{0}\right)^{2}}+O\left(\left|t-t_{0}\right|^{2}\right.$ ) for small $\left|t-t_{0}\right|$. Then the LandauZener formula states that there are corrections to (1.2) of exponential order $e^{-\pi \delta^{2} / 2 a \varepsilon}$.

It is notoriously difficult to prove the validity of such infinite order corrections in singular perturbation problems, and we are unaware of any rigorous proof of the Landau-Zener formula under general hypotheses on the Hamiltonian $H(t)$. However, exponentially small bounds on the correction terms have been obtained in some circumstances $[2,3,7,14]$.

In this paper, we consider a much less difficult problem. We prove the Landau-Zener formula for situations in which the minimum gap width depends on $\varepsilon$ and behaves like $c \varepsilon^{1 / 2}+O(\varepsilon)$. We further assume that the small gap arises from an avoided crossing of two isolated multiplicity one eigenvalues that are remain bounded an $O(1)$ distance away from the rest of the spectrum.

By an avoided crossing, one usually means a situation in which the gap between two isolated eigenvalues has a small, but positive local minimum. In this paper, we use the term avoided crossing to mean the following:

Definition. Suppose $H(t, \delta)$ is a family of self-adjoint operators with a fixed domain $\mathscr{D}$ in a Hilbert space $\mathscr{H}$. Suppose that resolvent of $H(t, \delta)$ is $C^{3}$ as an operator from $\mathscr{H}$ to $\mathscr{D}$ for $(t, \delta)$ in the domain $[-T, T] \times[-\alpha, \alpha]$. Suppose $H(t, \delta)$ has two isolated, multiplicity one eigenvalues $E_{\mathscr{A}}(t, \delta)$ and $E_{\not B}(t, \delta)$ for $(t, \delta) \neq\left(t_{0}, 0\right)$. Assume $E_{\mathscr{A}}(t, \delta)$ and $E_{\mathscr{B}}(t, \delta)$ are bounded away from the rest of the spectrum of $H(t, \delta)$ for all $t$ and $\delta$. Then if $E_{\mathscr{A}}\left(t_{0}, 0\right)=E_{\mathscr{B}}\left(t_{0}, 0\right)$, we say $H(t, \delta)$ has an avoided crossing between these two eigenvalues at $t_{0}$.

In Sect. 2 we discuss degenerate perturbation theory in two variables, and define what we mean by non-degenerate avoided crossings. The definition of non-degeneracy is technical. It is a non-vanishing condition for certain matrix elements of two $2 \times 2$ matrices that depend on the behavior of the eigenvalues of $H(t, \delta)$ and their spectral subspaces near the point $\left(t_{0}, 0\right)$. Avoided crossings generically satisfy this non-degeneracy condition.

The following proposition describes the local behavior of eigenvalues involved in a non-degenerate avoided crossing.

Proposition 1.1. Suppose $H(t, \delta)$ has a non-degenerate avoided crossing between two eigenvalues $E_{. S}(t, \delta)$ and $E_{\mathscr{B}}(t, \delta)$ at $t_{0}$, and assume (without loss) that $E_{. \delta}(t, \delta)>E_{\mathscr{B}}(t, \delta)$ 
for $\delta>0$. Then there exist real numbers $a>0, b \neq 0$, and $c>0$, such that

$$
E_{\mathscr{A}}(t, \delta)-E_{\mathscr{R}}(t, \delta)=2 \sqrt{a^{2} \tilde{t}^{2}+c^{2} \delta^{2}}+O\left(\tilde{t}^{2}+\delta^{2}\right),
$$

where $\tilde{t}=t-t_{0}+b \delta / a$.

We now state our main result. An analogous result holds if the roles of $E_{\mathscr{A}}$ and $E_{\mathscr{B}}$ are reversed.

Theorem 1.2. Suppose $H(t, \delta)$ has a single avoided crossing between two eigenvalues $E_{\mathscr{A}}(t, \delta)$ and $E_{\mathscr{O}}(t, \delta)$ on the interval $[-T, T]$ at some time $t_{0} \in(-T, T)$. Assume this avoided crossing is non-degenerate and that $E_{\mathscr{A}}(t, \delta)>E_{\mathscr{B}}(t, \delta)$ for $\delta>0$. Let $a, b$, and $c$ be the three numbers associated with this avoided crossing by Proposition 1.1. Then for $\left|t-t_{0}\right|>\beta>0$, one can choose $\Phi_{\mathscr{A}}(t, \delta)$ and $\Phi_{\mathscr{B}}(t, \delta)$ to be smooth normalized eigenvectors that correspond to $E_{\mathscr{A}}(t, \delta)$ and $E_{\mathscr{B}}(t, \delta)$, respectively, such that $\frac{\partial \Phi_{\mathscr{\ell}}}{\partial t}(t, \delta)$ is orthogonal to $\Phi_{\mathscr{G}}(t, \delta)$ for $\mathscr{C}=\mathscr{A}, \mathscr{B}$. Moreover, there is a solution to the Schrödinger equation

$$
i \varepsilon \frac{\partial \psi}{\partial t}=H\left(t, \varepsilon^{1 / 2}\right) \psi
$$

that satisfies

$$
\psi(t, \varepsilon)=\exp \left\{-i \int_{t_{0}-(b / a) \varepsilon^{1 / 2}}^{t} E_{\mathscr{A}}\left(r, \varepsilon^{1 / 2}\right) d r / \varepsilon\right\} \Phi_{\mathscr{A}}\left(t, \varepsilon^{1 / 2}\right)+O(\varepsilon)
$$

for $t \leqq t_{0}-\beta$. For $t \geqq t_{0}+\beta$, this solution satisfies

$$
\begin{aligned}
\psi(t, \varepsilon)= & \Lambda_{\mathscr{A}} \exp \left\{-i \int_{t_{0}-(b / a) \varepsilon^{1 / 2}}^{t} E_{\mathscr{A}}\left(r, \varepsilon^{1 / 2}\right) d r / \varepsilon\right\} \Phi_{\mathscr{A}}\left(t, \varepsilon^{1 / 2}\right) \\
& +\Lambda_{\mathscr{A}} \exp \left\{-i \int_{t_{0}-(b / a) \varepsilon^{1 / 2}}^{t} E_{\mathscr{B}}\left(r, \varepsilon^{1 / 2}\right) d r / \varepsilon\right\} \Phi_{\mathscr{B}}\left(t, \varepsilon^{1 / 2}\right) \\
& +O\left(\varepsilon^{p}\right),
\end{aligned}
$$

for some positive $p$,

$$
\Lambda_{\mathscr{A}}=\frac{(1-i) c}{2 a^{1 / 2}} \frac{\sqrt{2 \pi}}{\Gamma\left(1-\frac{i c^{2}}{2 a}\right)} \exp \left\{\frac{i c^{2}}{2 a}\left(1-\log \left(\frac{c^{2}}{2 a}\right)\right)\right\} e^{-\pi c^{2} / 4 a},
$$

and

$$
\Lambda_{\mathscr{B}}=e^{i \omega(\varepsilon)} e^{-\pi c^{2} / 2 a}
$$

Remarks. 1. Expressions (1.4)-(1.7) are the Landau-Zener formula for Eq. (1.3). Thus, the final conclusion to the theorem states that the formula is valid for non-degenerate avoided crossings.

2. Because we have choosen $\delta=\varepsilon^{1 / 2}$ in (1.3), the coefficients $\Lambda_{\mathscr{A}}$ and $\Lambda_{\mathscr{B}}$ that describe the mixing of the adiabatic states associated with $E_{\mathscr{A}}(t, \delta)$ and $E_{\mathscr{B}}(t, \delta)$ both are $O(1)$. If, instead, $\delta$ is held fixed as $\varepsilon \rightarrow 0$, then one expects $\Lambda_{\mathscr{B}}=e^{i \omega(\varepsilon)} e^{-\pi c^{2} \delta^{2} / 2 a \varepsilon}$ to be exponentially small. 
3. The analysis of exponentially small terms in singular perturbation theory is mathematically very interesting and difficult. By choosing $\delta=\varepsilon^{1 / 2}$ we have avoided these infinite order considerations. However, the limit we have chosen is appropriate for many physically interesting systems in which the propagation is approximately adiabatic and there are avoided crossings with small gaps. For example, the electronic propagation is adiabatic in many molecular systems, and such systems exhibit a wide variety of gap sizes at avoided crossings of eigenvalues. Another such example involves solar neutrino oscillations.

4. The proof of Theorem 1.2 involves matched asymptotic expansions. Expression (1.4) describes a negative time "outer solution." In an $\varepsilon$-dependent neighborhood of $t_{0},(1.4)$ is matched to an "inner solution" that depends on a rescaled time parameter $s=a^{1 / 2} \varepsilon^{-1 / 2} \tilde{t}$. This inner solution is then matched to a positive time outer solution that is described by (1.5)-(1.7).

5. Zener's original paper [16] only analyzed a special case in which $H(t)$ was a particular $2 \times 2$ matrix valued function. He argued physically that this special case reflected the behavior of general systems. Our proof shows that his intuition was correct. His physical reasoning led him to write down our inner solution. All the interesting mixing of the two adiabatic states occurs in the temporal boundary layer during which the inner solution describes the propagation.

6. In addition, Zener tacitly took the same type of limit that we discuss in this paper. In the middle of this analysis, he used asymptotics of parabolic cylinder functions that are valid when the eigenvalue gap has width $O\left(\varepsilon^{1 / 2}\right)$.

7. Landau's original paper [8] contained the beautiful idea that avoided crossings could be understood by analytic continuation to complex time. An avoided crossing was associated with an actual crossing at some point in the complex time plane. We have not seen a rigorous justification of the Landau-Zener formula from this point of view, although exponentially small bounds have been obtained this way $[7,14]$.

8. Except when symmetries are involved, avoided crossings should generically occur in adiabatic quantum mechanics rather than actual crossings. If there are no symmetries involved, crossings of eigenvalues of real symmetric or self-adjoint Hamiltonians generically occur on submanifolds of codimension 2 or 3 , respectively. Since $H(t)$ depends on only one parameter, $t$, one should not expect to see actual crossings unless symmetries are involved.

9. Although (1.6) appears to be complicated, its absolute square is not. The transition probabilities associated with the avoided crossing are

and

$$
\left|\Lambda_{\mathscr{A}}\right|^{2}=1-e^{-\pi c^{2} / a}
$$

$$
\left|\Lambda_{\mathscr{B}}\right|^{2}=e^{-\pi c^{2} / a} .
$$

10. The phase factor $e^{i \omega(\varepsilon)}$ that occurs in (1.7) depends on the choices of the phases of $\Phi_{\mathscr{A}}$ and $\Phi_{\mathscr{B}}$. Since we have only specified the choices up to an arbitrary time-independent phase factor, the value of $\omega(\varepsilon)$ is not determined. For any given choice of the eigenvector phases, $\omega(\varepsilon)$ is determined uniquely by the formulas in Sect. 3. 
The paper is organized as follows. Section 2 is devoted to a description of degenerate perturbation theory in two variables. What we mean by non-degenerate avoided crossings is precisely defined, and Proposition 1.1 is proved. Section 3 contains the proof of Theorem 1.2. It involves formal asymptotic expansions, some simple estimates, and the use of a lemma that is based on the fundamental theorem of calculus.

There is a huge physics literature associated with the Landau-Zener formula, and we will not attempt to review it here. We recommend the interested reader consult the introduction to the recent preprint of Jakšić and Segert [7].

\section{Degenerate Perturbation Theory in Two Valuables}

In this section, we discuss the structure of non-degenerate avoided crossings. This amounts to a study of degenerate perturbation theory for Hamiltonians that depend on two parameters.

Suppose two eigenvalues, $E_{\mathscr{A}}(t, \delta)$ and $E_{\mathscr{B}}(t, \delta)$, of $H(t, \delta)$ have an avoided crossing at time $t_{0}$ (and recall that by our definition, this requires the resolvent of $H(t, \delta)$ to be $C^{3}$ in $(t, \delta)$ as an operator from $\mathscr{H}$ to $\left.\mathscr{D}\right)$. We begin our analysis by letting $P(t, \delta)$ be the rank two orthogonal projection onto the spectral subspace of $H(t, \delta)$ that corresponds to these two eigenvalues. By representing $P(t, \delta)$ as a contour integral of the resolvent, it is easy to see that it is a $C^{3}$ operator-valued function. From this it follows that the trace of $H(t, \delta) P(t, \delta)$ (which equals $E_{\mathscr{A}}(t, \delta)+E_{\mathscr{B}}(t, \delta)$ ) is a $C^{3}$ real valued function. So, the resolvent of

$$
H_{1}(t, \delta)=H(t, \delta)-\frac{1}{2}\left(E_{\mathscr{A}}(t, \delta)+E_{\mathscr{B}}(t, \delta)\right)
$$

is also a $C^{3}$ operator-valued function. Clearly $H(t, \delta)$ and $H_{1}(t, \delta)$ have the same spectral projections, and the restriction of $H_{1}(t, \delta)$ to the range of $P(t, \delta)$ is traceless.

We choose $\left\{\psi_{1}, \psi_{2}\right\}$ to be an orthonormal basis for the range of $P\left(t_{0}, 0\right)$, and define

$$
\Psi_{1}(t, \delta)=\frac{P(t, \delta) \psi_{1}}{\sqrt{\left\langle\psi_{1}, P(t, \delta) \psi_{1}\right\rangle}} .
$$

Since $P(t, \delta)$ is $C^{3}$, it is easy to see that $\Psi_{1}(t, \delta)$ is a $C^{3}$ function of $t$ and $\delta$ in some neighborhood of $\left(t_{0}, 0\right)$.

Let $P_{1}(t, \delta)$ denote the orthogonal projection onto the subspace spanned by $\Psi_{1}(t, \delta)$. It is a $C^{3}$ operator-valued function in some neighborhood of $\left(t_{0}, 0\right)$, and

$$
P(t, \delta) P_{1}(t, \delta)=P_{1}(t, \delta) P(t, \delta)=P_{1}(t, \delta) .
$$

We define

$$
\Psi_{2}(t, \delta)=\frac{P(t, \delta)\left(1-P_{1}(t, \delta)\right) \psi_{2}}{\sqrt{\left\langle\psi_{2}, P(t, \delta)\left(1-P_{1}(t, \delta)\right) \psi_{2}\right\rangle}} .
$$

This vector-valued function is also $C^{3}$ in a neighborhood of $\left(t_{0}, 0\right)$, and $\left\{\Psi_{1}(t, \delta), \Psi_{2}(t, \delta)\right\}$ is an orthonormal basis for the range of $P(t, \delta)$ for $(t, \delta)$ in a neighborhood of $\left(t_{0}, 0\right)$.

Near $\left(t_{0}, 0\right)$, the restriction of $H_{1}(t, \delta)$ to the range of $P(t, \delta)$ is given in this basis 
by a traceless $2 \times 2$ matrix-valued function that is $C^{3}$ in $(t, \delta)$ and is the zero matrix at the $\left(t_{0}, 0\right)$. By standard Taylor series results, this matrix-valued function has the form

$$
A\left(t-t_{0}\right)+B \delta+O\left(\left|t-t_{0}\right|^{2}+\delta^{2}\right),
$$

where $A$ and $B$ are self-adjoint and traceless.

Non-Degeneracy Assumption I. We assume that $A$ is not the zero matrix.

By the spectral theorem applied to $A$, there is an orthonormal basis $\left\{\phi_{1}(t, \delta), \phi_{2}(t, \delta)\right\}$ that consists of $t$ - and $\delta$-independent linear combinations of $\left\{\Psi_{1}(t, \delta), \Psi_{2}(t, \delta)\right\}$, such that in the new basis, the restriction of $H_{1}(t, \delta)$ to the range of $P(t, \delta)$ is given near $\left(t_{0}, 0\right)$ by

$$
A_{1}\left(t-t_{0}\right)+B_{1} \delta+O\left(\left|t-t_{0}\right|^{2}+\delta^{2}\right)
$$

where $A_{1}$ is a diagonal matrix $\left(\begin{array}{cc}a & 0 \\ 0 & -a\end{array}\right)$ with $a>0$.

Non-Degeneracy Assumption II. We assume that the matrix $B_{1}$ is not a diagonal matrix (and hence, that it is non-zero).

Since $B_{1}$ is traceless and self-adjoint, it has the form $\left(\begin{array}{cc}b & \bar{c} \\ c & -b\end{array}\right)$, where $b$ is real
$c$ is complex and non-zero.

We define $\tilde{t}=t-t_{0}+\frac{b}{a} \delta$. Then in the basis $\left\{\phi_{1}(t, \delta), \phi_{2}(t, \delta)\right\}$, the restriction of $H_{1}(t, \delta)$ to the range of $P(t, \delta)$ is locally given by

$$
M_{1}(\tilde{t}, \delta)=\left(\begin{array}{cc}
a \tilde{t}+F_{1}(\tilde{t}, \delta) & \bar{c} \delta+\overline{F_{2}(\tilde{t}, \delta)} \\
c \delta+F_{2}(\tilde{t}, \delta) & -a \tilde{t}-F_{1}(\tilde{t}, \delta)
\end{array}\right),
$$

where the $F_{j}$ are $C^{3}, F_{j}(0,0)=0, \frac{\partial F_{j}}{\partial \tilde{t}}(0,0)=0, \frac{\partial F_{j}}{\partial \delta}(0,0)=0$, and $F_{1}$ is real. By multiplying $\phi_{2}(t, \delta)$ by a $t$ and $\delta$ dependent phase, we can assume without loss that $F_{2}$ is real and $c$ is real and positive.

Thus, Non-degeneracy Assumptions I and II guarantee that near $\left(t_{0}, 0\right)$, there is a $C^{3}$ basis,

$$
\left\{\phi_{1}(t, \delta), \phi_{2}(t, \delta)\right\}
$$

in which $H_{1}(t, \delta)$ is represented on $\mathscr{H} \cong \mathbb{C} \oplus \mathbb{C} \oplus(1-P(t, \delta)) \mathscr{H}$ as

$$
\left(\begin{array}{ccc}
a \tilde{t}+F_{1}(\tilde{t}, \delta) & c \delta+F_{2}(\tilde{t}, \delta) & 0 \\
c \delta+F_{2}(\tilde{t}, \delta) & -a \tilde{t}-F_{1}(\tilde{t}, \delta) & 0 \\
0 & 0 & H_{1}^{\perp}(\tilde{t}, \delta)
\end{array}\right)
$$

where $H_{1}^{\perp}(\tilde{t}, \delta)$ is the restriction of $H_{1}(t, \delta)$ to the range $(1-P(t, \delta))$. We note that $H_{1}^{\perp}(\tilde{t}, \delta)$ has a $C^{3}$ resolvent and has its spectrum bounded away from the spectrum of $M_{1}(\tilde{t}, \delta)$ for $(t, \delta)$ near $\left(t_{0}, 0\right)$.

From the representation (2.3), elementary linear algebra, standard Taylor series estimates, and tedious calculations, we obtain several results for $(t, \delta)$ in a 
neighborhood of $\left(t_{0}, \delta\right)$. We conclude this section with a list of these results, and note that the first of these is the conclusion to Proposition 1.1,

$$
\begin{gathered}
E_{\mathscr{A}}(t, \delta)-E_{\mathscr{B}}(t, \delta)=2 \sqrt{a^{2} \tilde{t}^{2}+c^{2} \delta^{2}}+O\left(\tilde{t}^{2}+\delta^{2}\right), \\
\frac{\partial}{\partial t}\left(E_{\mathscr{A}}-E_{\mathscr{B}}\right)(t, \delta)=2 \frac{a^{2} \tilde{t}}{\sqrt{a^{2} \tilde{t}^{2}+c^{2} \delta^{2}}}+O\left(\left(\tilde{t}^{2}+\delta^{2}\right)^{1 / 2}\right), \\
\Phi_{\mathscr{A}}(t, \delta)=e^{i \omega_{\mathscr{A}}(t, \delta)}\left(\cos (\theta / 2) \phi_{1}(t, \delta)+\sin (\theta / 2) \phi_{2}(t, \delta)\right),
\end{gathered}
$$

and

$$
\Phi_{\mathscr{B}}(t, \delta)=e^{i \omega_{\mathscr{S}}(t, \delta)}\left(-\sin (\theta / 2) \phi_{1}(t, \delta)+\cos (\theta / 2) \phi_{2}(t, \delta)\right),
$$

where $\omega_{\mathscr{A}}(t, \delta)$ and $\omega_{\mathscr{B}}(t, \delta)$ are functions to be chosen in Sect. 3 and

$$
\theta=\tan ^{-1}\left(\frac{c \delta+F_{2}(\tilde{t}, \delta)}{a \tilde{t}+F_{1}(\tilde{t}, \delta)}\right) .
$$

We only use $\theta$ for small $\delta>0$, and we choose $0<\theta<\pi$.

If $|a \tilde{t}|>c \delta>0$, we have

$$
\theta=\tan ^{-1}\left(\frac{c \delta}{a \tilde{t}}\right)+O(|\tilde{t}|)
$$

and in general for $\delta>0$, we have

$$
\frac{\partial \theta}{\partial \tilde{t}}=\frac{-a c \delta}{a^{2} \tilde{t}^{2}+c^{2} \delta^{2}}+O(1),
$$

and

$$
\frac{\partial^{2} \theta}{\partial \tilde{t}^{2}}=\frac{2 a^{3} c \tilde{t} \delta}{\left(a^{2} \tilde{t}^{2}+c^{2} \delta^{2}\right)^{2}}+O\left(\left(\tilde{t}^{2}+\delta^{2}\right)^{-1 / 2}\right) .
$$

\section{Proof of Theorem 1.2}

We begin this section with Lemma 3.1, whose proof is a simple application of the fundamental theorem of calculus. We then explicitly construct the inner and two outer approximate solutions. Theorem 1.2 follows from some simple estimates and three applications of Lemma 3.1.

Lemma 3.1. For each fixed value of $\varepsilon$, suppose that $K\left(r, \varepsilon^{1 / 2}\right)$ is a family of self-adjoint operators with a fixed domain $\mathscr{D}$, and assume that the resolvent of $K\left(r, \varepsilon^{1 / 2}\right)$, as an operator from $\mathscr{H}$ to $\mathscr{D}$, is a $C^{2}$ function of $r$. Suppose $\psi(r, \varepsilon)$ belongs to $\mathscr{D}$, is continuously differentiable in $r$, and approximately solves the Schrödinger equation in the sense that

$$
i \varepsilon^{\nu} \frac{\partial \psi}{\partial r}(r, \varepsilon)=K\left(r, \varepsilon^{1 / 2}\right) \psi(r, \varepsilon)+\zeta(r, \varepsilon)
$$

where $\zeta(r . \varepsilon)$ satisfies

$$
\|\zeta(r, \varepsilon)\| \leqq \mu(r, \varepsilon)
$$


on the time interval of interest. Suppose $\Psi(r, \varepsilon)$ is the exact solution to the Schrödinger equation with initial condition $\Psi\left(r_{0}, \varepsilon\right)=\psi\left(r_{0}, \varepsilon\right)$. Then the following estimate holds:

$$
\|\Psi(r, \varepsilon)-\psi(r, \varepsilon)\| \leqq \varepsilon^{-v} \int_{r_{0}}^{r} \mu\left(r^{\prime}, \varepsilon\right) d r^{\prime}
$$

Proof. Our hypotheses guarantee $[11,13,15]$ the existence of a strongly differentiable unitary propagator $U\left(r, r_{0}, \varepsilon\right)$ for the Schrödinger equation with timedependent Hamiltonian $K\left(r, \varepsilon^{1 / 2}\right)$. By the unitarity of this propagator and the fundamental theorem of calculus, the quantity on the left-hand side of (3.1) can be estimated as follows:

$$
\begin{aligned}
& \left\|U\left(r, r_{0}, \varepsilon\right) \psi\left(r_{0}, \varepsilon\right)-\psi(r, \varepsilon)\right\| \\
& =\left\|\psi\left(r_{0}, \varepsilon\right)-U\left(r_{0}, r, \varepsilon\right) \psi(r, \varepsilon)\right\| \\
& \quad \leqq\left\|\int_{r_{0}}^{r} \frac{\partial}{\partial r^{\prime}}\left(\psi\left(r_{0}, \varepsilon\right)-U\left(r_{0}, r^{\prime}, \varepsilon\right) \psi\left(r^{\prime}, \varepsilon\right)\right) d r^{\prime}\right\| \\
& \quad=\left\|\int_{r_{0}}^{r}\left(-i \varepsilon^{-v} U\left(r_{0}, r^{\prime}, \varepsilon\right) K\left(r^{\prime}, \varepsilon^{1 / 2}\right) \psi\left(r^{\prime}, \varepsilon\right)-U\left(r_{0}, r^{\prime}, \varepsilon\right) \frac{\partial \psi}{\partial r^{\prime}}\left(r^{\prime}, \varepsilon\right)\right) d r^{\prime}\right\| \\
& =\left\|\int_{r_{0}}^{r} i \varepsilon^{-v} U\left(r_{0}, r^{\prime}, \varepsilon\right) \zeta\left(r^{\prime}, \varepsilon\right) d r^{\prime}\right\| \\
& \quad \leqq \varepsilon^{-v} \int_{r_{0}}^{r} \mu\left(r^{\prime}, \varepsilon\right) d r^{\prime} .
\end{aligned}
$$

This proves the lemma.

3A. The Negative Time Outer Solution. To construct and prove the validity of the negative time outer solution, we mimic the construction and proof of Sect. 2 of [6].

We first comment that under the hypotheses of Theorem 1.2, we can easily make $C^{3}$ choices for the normalized eigenvector $e^{i \theta(t, \delta)} \hat{\Phi}_{s t}(t, \delta)$ for $\left|t-t_{0}\right|>R$, where $\theta(t, \delta)$ is an arbitrary real $C^{3}$ function. By differentiating the normalization condition

$$
\left\langle\hat{\Phi}_{\mathscr{A}}(t, \delta), \hat{\Phi}_{\mathscr{A}}(t, \delta)\right\rangle=1
$$

with respect to $t$, we learn that

$$
\left\langle\widehat{\Phi}_{\mathscr{A}}(t, \delta), \frac{\partial}{\partial t} \hat{\Phi}_{\mathscr{A}}(t, \delta)\right\rangle
$$

is purely imaginary. Thus, there are real solutions to

$$
i \frac{\partial \theta}{\partial t}=\left\langle\hat{\Phi}_{\mathscr{A}}(t, \delta), \frac{\partial}{\partial t} \hat{\Phi}_{\mathscr{A}}(t, \delta)\right\rangle .
$$

If we choose such a real solution $\theta(t, \delta)$ that depends smoothly on $\delta$, then

$$
\Phi_{\mathscr{A}}(t, \delta)=e^{i \theta(t, \delta)} \widehat{\Phi}_{\mathscr{A}}(t, \delta)
$$

satisfies

$$
\left\langle\Phi_{\mathscr{A}}(t, \delta), \frac{\partial}{\partial t} \Phi_{\mathscr{A}}(t, \delta)\right\rangle=0
$$


We choose $\Phi_{\mathscr{B}}(t, \delta)$ by the same technique, and note that these choices uniquely determine the functions $\omega_{\mathscr{A}}(t, \delta)$ and $\omega_{\mathscr{B}}(t, \delta)$ in Sect. 2 up to additive constants.

We next make the ansatz that (1.3) has a formal solution of the form

$$
\begin{aligned}
\psi(t, \varepsilon)= & \exp \left\{-i \int_{t_{0}-(b / a) \varepsilon^{1 / 2}}^{t} E_{\mathscr{A}}\left(r, \varepsilon^{1 / 2}\right) d r / \varepsilon\right\} \\
& \cdot\left(\psi_{0}\left(t, \varepsilon^{1 / 2}\right)+\varepsilon \psi_{1}\left(t, \varepsilon^{1 / 2}\right)+\varepsilon^{2} \psi_{2}\left(t, \varepsilon^{1 / 2}\right)+\cdots\right)
\end{aligned}
$$

for $t<t_{0}-\beta$.

We substitute (3.2) into (1.3) and equate terms of like powers of $\varepsilon$ on the two sides of the resulting equation.

The zeroth order terms require

$$
H\left(t, \varepsilon^{1 / 2}\right) \psi_{0}\left(t, \varepsilon^{1 / 2}\right)=E_{\mathscr{A}}\left(t, \varepsilon^{1 / 2}\right) \psi_{0}\left(t, \varepsilon^{1 / 2}\right) .
$$

Thus,

$$
\psi_{0}\left(t, \varepsilon^{1 / 2}\right)=f_{0}(t, \varepsilon) \Phi_{\mathscr{A}}\left(t, \varepsilon^{1 / 2}\right)
$$

where $f_{0}$ is not yet determined.

The first order terms require

$$
i \frac{\partial f_{0}}{\partial t}\left(t, \varepsilon^{1 / 2}\right) \Phi_{\mathscr{A}}\left(t, \varepsilon^{1 / 2}\right)+i f_{0}\left(t, \varepsilon^{1 / 2}\right) \frac{\partial \Phi_{\mathscr{A}}}{\partial t}\left(t, \varepsilon^{1 / 2}\right)=\left[H\left(t, \varepsilon^{1 / 2}\right)-E_{\mathscr{A}}\left(t, \varepsilon^{1 / 2}\right)\right] \psi_{1}\left(t, \varepsilon^{1 / 2}\right) .
$$

We split this into two conditions by separately considering components of this equation that are multiples of $\Phi_{\mathscr{A}}\left(t, \varepsilon^{1 / 2}\right)$, and those are orthogonal to $\Phi_{\mathscr{A}}\left(t, \varepsilon^{1 / 2}\right)$ :

$$
i \frac{\partial f_{0}}{\partial t}\left(t, \varepsilon^{1 / 2}\right)=0
$$

and

$$
i f_{0}\left(t, \varepsilon^{1 / 2}\right) \frac{\partial \Phi_{\mathscr{A}}}{\partial t}\left(t, \varepsilon^{1 / 2}\right)=\left[H\left(t, \varepsilon^{1 / 2}\right)-E_{\mathscr{A}}\left(t, \varepsilon^{1 / 2}\right)\right] \psi_{1}\left(t, \varepsilon^{1 / 2}\right)
$$

Since we are only doing a leading order expansion, we terminate the expansion at this stage and choose the following particular solutions to these equations:

$$
f_{0}\left(t, \varepsilon^{1 / 2}\right)=1 \text {, }
$$

and

$$
\psi_{1}^{\perp}\left(t, \varepsilon^{1 / 2}\right)=i\left[H\left(t, \varepsilon^{1 / 2}\right)-E_{\mathscr{A}}\left(t, \varepsilon^{1 / 2}\right)\right]_{r}^{-1} \frac{\partial \Phi_{\mathscr{A}}}{\partial t}\left(t, \varepsilon^{1 / 2}\right),
$$

where $\left[H\left(t, \varepsilon^{1 / 2}\right)-E_{\mathscr{A}}\left(t, \varepsilon^{1 / 2}\right)\right]_{r}^{-1}$ denotes the restriction of the resolvent of $H\left(t, \varepsilon^{1 / 2}\right)$. to the subspace orthogonal to $\Phi_{\mathscr{A}}\left(t, \varepsilon^{1 / 2}\right)$.

We define the negative time outer approximate solution to be

$$
\psi_{\mathscr{A}}(t, \varepsilon)=\exp \left\{-i \int_{t_{0}-(b / a) \varepsilon^{1 / 2}}^{t} E_{\mathscr{A}}\left(r, \varepsilon^{1 / 2}\right) d r / \varepsilon\right\}\left(\Phi_{\mathscr{A}}\left(t, \varepsilon^{1 / 2}\right)+\varepsilon \psi_{1}^{\perp}\left(t, \varepsilon^{1 / 2}\right)\right) .
$$

This expression approximately satisfies Eq. (1.3) in the sense of Lemma 3.1 with a remainder term

$$
\zeta(t, \varepsilon)=-i \varepsilon^{2} \exp \left\{-i \int_{t_{0}-(b / a) \varepsilon^{1 / 2}}^{t} E_{\mathscr{A}}\left(r, \varepsilon^{1 / 2}\right) d r / \varepsilon\right\} \frac{\partial \psi_{1}^{\perp}}{\partial t}\left(t, \varepsilon^{1 / 2}\right) .
$$


For $t \leqq t_{0}-\beta$, this error term has norm bounded by $C \varepsilon^{2}$, so Lemma 3.1 with $v=1$ and $r=t$ shows that (3.3) agrees with an exact solution to Eq. (1.3) up to an $O(\varepsilon)$ error for $t \leqq t_{0}-\beta$. Since (1.4) agrees with (3.3) on this time interval, there is a solution to (1.3) that satisfies (1.4) for $t \leqq t_{0}-\beta$.

As $t$ approaches $t_{0}$, the norm of (3.4) diverges. This norm is equal to the norm of

$$
\begin{aligned}
& \varepsilon^{2}\left(\frac{\partial}{\partial t}\left[H\left(t, \varepsilon^{1 / 2}\right)-E_{\mathscr{S}}\left(t, \varepsilon^{1 / 2}\right)\right]_{r}^{-1}\right) \frac{\partial \Phi_{\mathscr{A}}}{\partial t}\left(t, \varepsilon^{1 / 2}\right) \\
& \quad+\varepsilon^{2}\left[H\left(t, \varepsilon^{1 / 2}\right)-E_{s d}\left(t, \varepsilon^{1 / 2}\right)\right]_{r}^{-1} \frac{\partial^{2} \Phi_{\mathscr{A}}}{\partial t^{2}}\left(t, \varepsilon^{1 / 2}\right) .
\end{aligned}
$$

We estimate how this norm diverges with the help of formulas (2.4)-(2.11). From (2.6) and (2.10), it follows by explicit calculation, that the norm of the final factor in the first term in (3.5) is bounded by a constant times

$$
\left|\frac{\partial \theta}{\partial t}\right|=\frac{a c \varepsilon^{1 / 2}}{a^{2} \tilde{t}^{2}+c^{2} \varepsilon} .
$$

By (2.4), (2.6), (2.10), (2.11), and explicit calculations, the norm of the second term in (3.5) is obtained by a constant times

$$
\varepsilon^{2} \frac{1}{2 \sqrt{a^{2} \tilde{t}^{2}+c^{2} \varepsilon}} \frac{a^{3} c \varepsilon^{1 / 2}|\tilde{t}|+a^{2} c^{2} \varepsilon}{\left(a^{2} \tilde{t}^{2}+c^{2} \varepsilon\right)^{2}} .
$$

To estimate the operator norm of the middle factor in the first term of (3.5), we write the reduced resolvent as

$$
\begin{aligned}
{[H(t,} & \left.\left.\varepsilon^{1 / 2}\right)-E_{\mathscr{A}}\left(t, \varepsilon^{1 / 2}\right)\right]_{r}^{-1} \\
= & \left(E_{\mathscr{B}}\left(t, \varepsilon^{1 / 2}\right)-E_{\mathscr{S}}\left(t, \varepsilon^{1 / 2}\right)\right)^{-1}\left|\Phi_{\mathscr{B}}\left(t, \varepsilon^{1 / 2}\right)\right\rangle\left\langle\Phi_{\mathscr{B}}\left(t, \varepsilon^{1 / 2}\right)\right| \\
& \quad+\left[H_{1}^{\perp}\left(\tilde{t}, \varepsilon^{1 / 2}\right)-\frac{1}{2}\left(E_{\mathscr{S}}\left(t, \varepsilon^{1 / 2}\right)-E_{\mathscr{B}}\left(t, \varepsilon^{1 / 2}\right)\right)\right]^{-1}\left(1-P\left(t, \varepsilon^{1 / 2}\right)\right) .
\end{aligned}
$$

The second term on the right-hand side of (3.6) has bounded derivative. To estimate the first term on the right-hand side, we use (2.7) and then explicitly differentiate. From (2.4), (2.5) and (2.10), we find that the operator norm of the derivative of (3.6) is bounded by a constant plus a constant times

$$
\frac{2 a|\tilde{t}|}{\left(a^{2} \tilde{t}^{2}+c^{2} \varepsilon\right)^{3 / 2}}+\frac{1}{\sqrt{a^{2} \tilde{t}^{2}+c^{2} \varepsilon}} \frac{a c \varepsilon^{1 / 2}}{\left(a^{2} \tilde{t}^{2}+c^{2} \varepsilon\right)} .
$$

By combining all these estimates, we find that the norm of (3.4) is bounded by a constant multiple of

$$
\begin{aligned}
& \varepsilon^{2}\left(\frac{\varepsilon^{1 / 2}|\tilde{t}|}{\left(a^{2} \tilde{t}^{2}+c^{2} \varepsilon\right)^{5 / 2}}+\frac{\varepsilon}{\left(a^{2} \tilde{t}^{2}+c^{2} \varepsilon\right)^{5 / 2}}+\frac{\varepsilon^{1 / 2}}{\left(a^{2} \tilde{t}^{2}+c^{2} \varepsilon\right)}\right) \\
& \quad \leqq C\left(\varepsilon^{5 / 2}|\tilde{t}|^{-4}+\varepsilon^{3}|\tilde{t}|^{-5}+\varepsilon^{5 / 2}|\tilde{t}|^{-2}\right) .
\end{aligned}
$$

So, by Lemma 3.1, (3.3) agrees with an exact solution to (1.3) up to an $O\left(\varepsilon^{(3 / 2)-3 \gamma}\right)$ as long as $\tilde{t} \leqq-\varepsilon^{\gamma}$. If we keep $\gamma<\frac{1}{2}$, this error is bounded by a positive power of $\varepsilon$.

3B. The Inner Solution. Near crossings, a new time scale becomes relevant (see 
[6]). Accordingly, we introduce a new time variable $s=a^{1 / 2} \varepsilon^{-1 / 2} \tilde{t}$. When necessary, we let $t(s, \varepsilon)=a^{-1 / 2} \varepsilon^{1 / 2} s+t_{0}-\frac{b}{a} \varepsilon^{1 / 2}$ denote the old time variable as a function of the new one. The rescaled version of Eq. (1.3) is

$$
i \varepsilon^{1 / 2} a^{-1 / 2} \frac{\partial \psi}{\partial s}=\tilde{H}\left(s, \varepsilon^{1 / 2}\right) \psi,
$$

where

$$
\tilde{H}\left(s, \varepsilon^{1 / 2}\right)=H\left(t(s, \varepsilon), \varepsilon^{1 / 2}\right) .
$$

We now search for a solution to (3.7) that matches the negative time outer solution (3.3) when $\tilde{t}$ is small and negative, but $s$ is large and negative.

We begin by proceeding formally. We make the ansatz that the inner solution has the form

$$
\begin{aligned}
& \exp \left\{-i \int_{t_{0}-(b / a) \varepsilon^{1 / 2}}^{t(s, \varepsilon)}\left(E_{\mathscr{A}}\left(r, \varepsilon^{1 / 2}\right)+E_{\mathscr{B}}\left(r, \varepsilon^{1 / 2}\right)\right) d r / 2 \varepsilon\right\} \\
& \quad\left(\left(f_{0}(s)+v_{1}(\varepsilon) f_{1}(s)+\cdots\right) \phi_{1}\left(t(s, \varepsilon), \varepsilon^{1 / 2}\right)\right. \\
& \quad+\left(g_{0}(s)+v_{1}(\varepsilon) g_{1}(s)+\cdots\right) \phi_{2}\left(t(s, \varepsilon), \varepsilon^{1 / 2}\right) \\
& \left.\quad+\psi_{0}(s)+v_{1}(\varepsilon) \psi_{1}(s)+\cdots\right)
\end{aligned}
$$

where the $v_{j}(\varepsilon)$ are arbitrary order functions, and the $\psi_{j}$ are orthogonal to the range of the rank two projection $P\left(t(s, \varepsilon), \varepsilon^{1 / 2}\right)$. Note that the $\phi_{j}$ here are not the eigenvectors, but the vectors constructed near the end of Sect. 2.

We substitute (3.8) into (3.7), use representation (2.3) with the $F_{j}$ expanded in their second order Taylor series with remainder, and equate terms of like powers of $\varepsilon$ on the two sides of the resulting equation.

The leading order terms have order $\varepsilon^{0}$. They require

$$
\left(1-P\left(t(s, \varepsilon), \varepsilon^{1 / 2}\right)\right) H_{1}^{\perp}\left(a^{-1 / 2} \varepsilon^{1 / 2} s, \varepsilon^{1 / 2}\right) \psi_{0}=0 .
$$

Since $P\left(t(s, \varepsilon), \varepsilon^{1 / 2}\right) \psi_{0}=0$, this forces us to take $\psi_{0}=0$.

If $\lim _{\varepsilon \rightarrow 0} \varepsilon^{-1 / 2} v_{1}(\varepsilon)$ is infinite, the next order terms similarly require only $\psi_{1}=0$. Since this is trivial, we assume $\lim _{\varepsilon \rightarrow 0} \varepsilon^{-1 / 2} v_{1}(\varepsilon)$ to be finite. Thus, $v_{1}(\varepsilon)=o\left(\varepsilon^{1 / 2}\right)$ or $v_{1}(\varepsilon)=\varepsilon^{1 / 2}$. In either case, the next order terms are of order $\varepsilon^{1 / 2}$. If $v_{1}(\varepsilon)=o\left(\varepsilon^{1 / 2}\right)$, the order $\varepsilon^{1 / 2}$ terms require

$$
i \frac{\partial}{\partial s}\left(\begin{array}{l}
f_{0} \\
g_{0}
\end{array}\right)=\left(\begin{array}{cc}
s & c a^{-1 / 2} \\
c a^{-1 / 2} & -s
\end{array}\right)\left(\begin{array}{l}
f_{0} \\
g_{0}
\end{array}\right) .
$$

If $v_{1}(\varepsilon)=\varepsilon^{1 / 2}$, the order $\varepsilon^{1 / 2}$ terms require a more complicated condition. However, by separately examining the components in this condition that are in the ranges of $\left.P\left(t(s, \varepsilon), \varepsilon^{1 / 2}\right)\right)$ and $\left(1-P\left(t(s, \varepsilon), \varepsilon^{1 / 2}\right)\right)$, we find that this condition splits into two separate conditions. One is (3.9); the other is

$$
\left(1-P\left(t(s, \varepsilon), \varepsilon^{1 / 2}\right)\right) H_{1}^{\perp}\left(a^{-1 / 2} \varepsilon^{1 / 2} s, \varepsilon^{1 / 2}\right) \psi_{1}=0 .
$$

This latter condition implies $\psi_{1}=0$.

Thus, if either $v_{1}(\varepsilon)=o\left(\varepsilon^{1 / 2}\right)$ or $v_{1}(\varepsilon)=\varepsilon^{1 / 2}$, Eq. (3.9) must be satisfied. The solutions to this equation can be expressed in terms of parabolic cylinder functions. 
As we discuss below, the particular solution that formally matches the outer solution (3.3) is the following:

where

$$
\left(\begin{array}{l}
f_{0} \\
g_{0}
\end{array}\right)=K\left(\begin{array}{c}
\frac{(1-i) c}{2 a^{1 / 2}} D_{\left(i c^{2} / 2 a\right)-1}((i-1) s) \\
D_{\left(i c^{2} / 2 a\right)}((i-1) s)
\end{array}\right)
$$

$$
K=\exp \left\{i \omega_{\mathscr{A}}\left(t_{0}-\frac{b}{a} \varepsilon^{1 / 2}, \varepsilon^{1 / 2}\right)\right\} \exp \left\{-\frac{i c^{2}}{4 a} \log \left(\frac{c^{2}}{2 a}\right)+\frac{i c^{2}}{4 a}-\frac{\pi c^{2}}{8 a}\right\} .
$$

In addition, the matching condition requires $\psi_{1}=0$, even in the case where $v_{1}(\varepsilon)=o\left(\varepsilon^{1 / 2}\right)$.

We terminate the formal construction at this stage since we are only doing a leading order calculation. Thus, we take the inner approximate solution to be

$$
\begin{aligned}
& \exp \left\{-i \int_{t_{0}-(b / a) \varepsilon^{1 / 2}}^{t(s, \varepsilon)}\left(E_{\mathscr{A}}\left(r, \varepsilon^{1 / 2}\right)+E_{\mathscr{B}}\left(r, \varepsilon^{1 / 2}\right)\right) d r / 2 \varepsilon\right\} \exp \left\{i \omega_{\mathscr{A}}\left(t_{0}-\frac{b}{a} \varepsilon^{1 / 2}, \varepsilon^{1 / 2}\right)\right\} \\
& \cdot \exp \left\{-\frac{i c^{2}}{4 a} \log \left(\frac{c^{2}}{2 a}\right)+\frac{i c^{2}}{4 a}-\frac{\pi c^{2}}{8 a}\right\} \\
& \cdot\left(\frac{(1-i) c}{2 a^{1 / 2}} D_{\left(i c^{2} / 2 a\right)-1}((i-1) s) \phi_{1}\left(t(s, \varepsilon), \varepsilon^{1 / 2}\right)+D_{\left(i c^{2} / 2 a\right)}((i-1) s) \phi_{2}\left(t(s, \varepsilon), \varepsilon^{1 / 2}\right)\right) .
\end{aligned}
$$

We now must prove that this agrees with an exact solution and that it matches the negative time outer solution. The first of these tasks is relatively easy. By explicit calculation using the representation (2.3), we see that (3.10) approximately satisfies (3.7) with a remainder $\zeta$ that contains two types of terms. One type comes from the error term in (2.3) and has norm bounded by a constant times $\varepsilon s^{2}+\varepsilon$. The other type contains $i \varepsilon^{1 / 2} \frac{\partial}{\partial s}$ applied to the basis vectors $\phi_{j}(t(s, \varepsilon), \varepsilon)$, and has norm bounded by a constant times $\varepsilon$. To obtain these estimates, we have used the boundedness of (3.10), which follows from the self-adjointness of the matrix in (3.9). Thus, (3.10) approximately solves (3.7) in the sense of Lemma 3.1 with a remainder $\zeta$ whose norm at time $s$ is bounded by a constant times $\varepsilon s^{2}+\varepsilon$. We apply Lemma 3.1 with $v=\frac{1}{2}$ and $r=s$. We conclude that (3.10) agrees with an exact solution to (3.7) up to an $O\left(\varepsilon^{3 \gamma^{\prime}-1}\right)$ error as long as we keep $|\tilde{t}|<\varepsilon^{\gamma^{\prime}}$. If we choose $\gamma^{\prime}>1 / 3$, the error is bounded by a positive power of $\varepsilon$.

We fix $1 / 3<\gamma^{\prime}<\gamma<1 / 2$. Then for $-\varepsilon^{\gamma^{\prime}}<\tilde{t}<-\varepsilon^{\gamma}$, both the negative time outer solution and the inner solution agree with exact solutions up to $O\left(\varepsilon^{p}\right)$ errors, for some $p>0$. We now must prove that they agree with one another up to such an error in this overlap region. To do this, we set $\tilde{t}=-\varepsilon^{\kappa}$, where $k \in\left(\gamma^{\prime}, \gamma\right)$ is arbitrary, and we compute both solutions modulo errors that are bounded by positive powers of $\varepsilon$.

For $\tilde{t}=-\varepsilon^{\kappa}$, the norm of $\varepsilon \psi_{1}^{\perp}\left(t, \varepsilon^{1 / 2}\right)$ in (3.3) is

$$
\varepsilon \frac{1}{\sqrt{a^{2} \varepsilon^{2 \kappa}+c^{2} \varepsilon}} \frac{a c \varepsilon^{1 / 2}}{a^{2} \varepsilon^{2 \kappa}+c^{2} \varepsilon} \leqq C \varepsilon^{(3 / 2)-3 \kappa} .
$$


Thus, in the matching region, we can ignore this term, and up to an $O\left(\varepsilon^{p}\right)$ error, (3.3) equals

$$
\begin{aligned}
& \exp \left\{-i \int_{t_{0}-(b / a) \varepsilon^{1 / 2}}^{t}\left(E_{\mathscr{A}}\left(r, \varepsilon^{1 / 2}\right)+E_{\mathscr{A}}\left(r, \varepsilon^{1 / 2}\right)\right) d r / 2 \varepsilon\right\} \\
& \cdot \exp \left\{-i \int_{t_{0}-(b / a) \varepsilon^{1 / 2}}^{t}\left(E_{\mathscr{A}}\left(r, \varepsilon^{1 / 2}\right)-E_{\mathscr{A}}\left(r, \varepsilon^{1 / 2}\right)\right) d r / 2 \varepsilon\right\} \Phi_{\mathscr{A}}\left(t, \varepsilon^{1 / 2}\right) \\
& =\exp \left\{-i \int_{t_{0}-(b / a) \varepsilon^{1 / 2}}^{t}\left(E_{\mathscr{A}}\left(r, \varepsilon^{1 / 2}\right)+E_{\mathscr{A}}\left(r, \varepsilon^{1 / 2}\right)\right) d r / 2 \varepsilon\right\} \\
& \cdot \exp \left\{i \omega_{\mathscr{A}}\left(t_{0}-\frac{b}{a} \varepsilon^{1 / 2}, \varepsilon^{1 / 2}\right)\right\} \Omega_{\mathscr{A}}(\tilde{t}, \varepsilon) .
\end{aligned}
$$

Using the basis $\left\{\phi_{1}, \phi_{2}\right\}$ and formula (2.4), we can write $\Omega_{\mathscr{A}}(\tilde{t}, \varepsilon)$ for $\tilde{t}=-\varepsilon^{\kappa}$, as

$$
\begin{aligned}
\Omega_{\mathscr{A}}(\tilde{t}, \varepsilon)= & \exp \left\{-i \int_{t_{0}-(b / a) \varepsilon^{1 / 2}}^{t}\left(E_{\mathscr{A}}\left(r, \varepsilon^{1 / 2}\right)-E_{\mathscr{A}}\left(r, \varepsilon^{1 / 2}\right)\right) d r / 2 \varepsilon\right\}\left(\begin{array}{c}
\cos (\theta / 2) \\
\sin (\theta / 2)
\end{array}\right), \\
= & \exp \left\{-\frac{i}{2 a \varepsilon}\left[-a \varepsilon^{\kappa} \sqrt{a^{2} \varepsilon^{2 \kappa}+c^{2} \varepsilon}+c^{2} \varepsilon \log \left(-a \varepsilon^{\kappa}+\sqrt{a^{2} \varepsilon^{2 \kappa}+c^{2} \varepsilon}\right)\right]\right\} \\
& \cdot \exp \left\{-\frac{i}{2 a \varepsilon}\left[-c^{2} \varepsilon \log \left(c \varepsilon^{1 / 2}\right)+O\left(\varepsilon^{3 \kappa}+\varepsilon^{1+\kappa}\right)\right]\right\}\left(\begin{array}{c}
\cos (\theta / 2) \\
\sin (\theta / 2)
\end{array}\right)
\end{aligned}
$$

where $\theta=\tan ^{-1}\left(c \varepsilon^{(1 / 2)-\kappa} / a\right)+O\left(\varepsilon^{\kappa}\right)$ by (2.9). To evaluate the small $\varepsilon$ asymptotics of (3.11), we use (2.9), standard Taylor series estimates, and

$$
\sqrt{a^{2} \varepsilon^{2 \kappa}+c^{2} \varepsilon}=a \varepsilon^{\kappa}+\frac{c^{2}}{2 a} \varepsilon^{1-\kappa}+O\left(\varepsilon^{2-3 \kappa}\right)
$$

to obtain

$$
\begin{aligned}
\Omega_{\mathscr{A}}(\tilde{t}, \varepsilon)= & \exp \left\{-\frac{i}{2 a}\left[-a^{2} \varepsilon^{2 \kappa-1}-\frac{c^{2}}{2}+O\left(\varepsilon^{1-2 \kappa}\right)+c^{2} \log \left(\frac{c^{2} \varepsilon^{1-\kappa}}{2 a}+O\left(\varepsilon^{2}-3 \kappa\right)\right)\right]\right\} \\
& \cdot \exp \left\{-\frac{i}{2 a}\left[-c^{2} \log \left(c \varepsilon^{1 / 2}\right)\right]\right\}\left(\begin{array}{l}
0 \\
1
\end{array}\right)+O\left(\varepsilon^{p}\right) \\
= & \exp \left\{\frac{i a \varepsilon^{2 \kappa-1}}{2}\right\} \exp \left\{\frac{i c^{2}}{4 a}\right\} \exp \left\{-\frac{i c^{2}}{2 a} \log \left(\frac{c \varepsilon^{(1 / 2)-\kappa}}{2 a}\right)\right\}\left(\begin{array}{l}
0 \\
1
\end{array}\right)+O\left(\varepsilon^{p}\right),
\end{aligned}
$$

for some $p>0$.

So, for $\tilde{t}=-\varepsilon^{\kappa}$, the outer solution (3.3) equals

$$
\begin{gathered}
\exp \left\{-i \int_{t_{0}-(b / a) \varepsilon^{1 / 2}}^{t}\left(E_{\mathscr{A}}\left(r, \varepsilon^{1 / 2}\right)+E_{\mathscr{B}}\left(r, \varepsilon^{1 / 2}\right)\right) d r / 2 \varepsilon\right\} \exp \left\{i \omega_{\mathscr{A}}\left(t_{0}-\frac{b}{a} \varepsilon^{1 / 2}, \varepsilon^{1 / 2}\right)\right\} \\
\cdot \exp \left\{\frac{i a \varepsilon^{2 \kappa-1}}{2}\right\} \exp \left\{\frac{i c^{2}}{4 a}\right\} \exp \left\{-\frac{i c^{2}}{2 a} \log \left(\frac{c \varepsilon^{(1 / 2)-\kappa}}{2 a}\right)\right\} \phi_{2}\left(t_{0}, \varepsilon^{1 / 2}\right)+O\left(\varepsilon^{p}\right) .
\end{gathered}
$$

To evaluate the asymptotics of the inner solution for $\tilde{t}=-\varepsilon^{\kappa}$ we note that $s=-a^{1 / 2} \varepsilon^{\kappa-(1 / 2)}$ and use the known asymptotics of the parabolic cylinder functions 
[5] for large complex arguments. These show that the $\phi_{1}$ component in (3.10) is $O\left(\varepsilon^{(1 / 2)-\kappa}\right)$. Thus, in the negative time matching region, the inner solution $(3.10)$ equals

$$
\begin{aligned}
& \exp \left\{-i \int_{t_{0}-(b / a) \varepsilon^{1 / 2}}^{t}\left(E_{s A}\left(r, \varepsilon^{1 / 2}\right)+E_{\mathscr{B}}\left(r, \varepsilon^{1 / 2}\right)\right) d r / 2 \varepsilon\right\} \exp \left\{i \omega_{\triangle A}\left(t_{0}-\frac{b}{a} \varepsilon^{1 / 2}, \varepsilon^{1 / 2}\right)\right\} \\
& \cdot \frac{(1-i) c}{2 a^{1 / 2}} e^{i c^{2} / 4 a} \Xi(\tilde{t}, \varepsilon) \phi_{2}\left(t(s, \varepsilon), \varepsilon^{1 / 2}\right)+O\left(\varepsilon^{p}\right),
\end{aligned}
$$

where

$$
\Xi(\tilde{t}, \varepsilon)=e^{i c^{2} / 4 a} \exp \left\{-\frac{i c^{2}}{4 a} \log \left(c^{2} / 2 a\right)\right\} e^{-\pi c^{2} / 8 a} D_{i c^{2} / 2 a}((i-1) s) .
$$

For $\tilde{t}=-\varepsilon^{\kappa}$,

$$
\begin{aligned}
\Xi(\tilde{t}, \varepsilon)= & e^{i c^{2} / 4 a} \exp \left\{-\frac{i c^{2}}{4 a} \log \left(c^{2} / 2 a\right)\right\} e^{-\pi c^{2} / 8 a} \\
& \cdot \exp \left\{\frac{i a \varepsilon^{2 \kappa-1}}{2}+\frac{i c^{2}}{2 \alpha} \log \left((1-i) a^{1 / 2} \varepsilon^{\kappa-(1 / 2)}\right)\right\}+O\left(\varepsilon^{p}\right) \\
= & \exp \left\{\frac{i a \varepsilon^{2 \kappa-1}}{2}\right\} e^{i c^{2} / 4 a} \exp \left\{-\left(\frac{i c^{2}}{2 a}\right) \log \left(\frac{c \varepsilon^{(1 / 2)-\kappa}}{2 \alpha}\right)\right\}+O\left(\varepsilon^{p}\right) .
\end{aligned}
$$

So, we see that the inner solution also agrees with (3.12) for $\tilde{t}=-\varepsilon^{\kappa}$, and the matching is justified.

3C. The Positive Time Outer Solution. The positive time outer solution is a linear combination of the two standard adiabatic states associated with $E_{S}$ and $E_{\mathscr{B}}$. The particular linear combination is determined by matching the inner solution.

To construct the two standard adiabatic states we use the same technique we used to construct (3.3). The state associated with $E_{s}$ is given by (3.3), except we now keep $t>t_{0}-\frac{b}{a} \varepsilon^{1 / 2}$. The state associated with $E_{\not B}$ is

$$
\psi_{\mathscr{B}}(t, \varepsilon)=\exp \left\{-i \int_{t_{0}-(b / a) \varepsilon^{1 / 2}}^{t} E_{\mathscr{B}}\left(r, \varepsilon^{1 / 2}\right) d r / \varepsilon\right\}\left(\Phi_{\mathscr{B}}\left(t, \varepsilon^{1 / 2}\right)+\varepsilon \psi_{1}^{\perp}\left(t, \varepsilon^{1 / 2}\right)\right),
$$

where in this instance, $\psi_{1}^{\perp}$ is the function

$$
\psi_{1}^{\perp}\left(t, \varepsilon^{1 / 2}\right)=i\left[H\left(t, \varepsilon^{1 / 2}\right)-E_{3 / B}\left(t, \varepsilon^{1 / 2}\right)\right]_{r}^{-1} \frac{\partial \Phi_{3 / ~}}{\partial t}\left(t, \varepsilon^{1 / 2}\right),
$$

where $\left[H\left(t, \varepsilon^{1 / 2}\right)-E_{3 \beta}\left(t, \varepsilon^{1 / 2}\right)\right]_{r}^{-1}$ denotes the restriction of the resolvent of $H\left(t, \varepsilon^{1 / 2}\right)$ to the subspace orthogonal to $\Phi_{\mathscr{B}}\left(t, \varepsilon^{1 / 2}\right)$.

These two standard adiabatic states agree with the exact solutions of (1.3) up to $O\left(\varepsilon^{(3 / 2)-3 \gamma}\right)$ errors as long as $\tilde{t} \geqq \varepsilon^{\gamma}$. The proof of this claim is essentially the same as the proof in Sect. $3 \mathrm{~A}$ that the negative time outer solution agrees with an exact solution up to an $O\left(\varepsilon^{(3 / 2)-3 \%}\right)$ error. 
We note that when $t>t_{0}+\beta,(1.5)$ agrees with

$$
\Lambda_{\mathscr{A}} \psi_{\mathscr{A}}\left(t, \varepsilon^{1 / 2}\right)+\Lambda_{\mathscr{B}} \psi_{\mathscr{B}}\left(t, \varepsilon^{1 / 2}\right)
$$

up to an $O\left(\varepsilon^{p}\right)$ error. So, the proof of Theorem 1.2 will be complete as soon as we check that the inner solution and (3.13) agree with one another up to an $O\left(\varepsilon^{p}\right)$ error when $\tilde{t}=\varepsilon^{\kappa}$, where $\gamma^{\prime}<\kappa<\gamma$. We check this by explicitly evaluating the asymptotics of (3.10) and (3.13) in the matching region.

The inner solution (3.10) has two components: multiples of $\phi_{1}$ and multiples of $\phi_{2}$. The factors that multiply $\phi_{1}$ in (3.10) are

$$
\begin{aligned}
\exp & \left\{-i \int_{t_{0}-(b / a) \varepsilon^{1 / 2}}^{t(s, \varepsilon)}\left(E_{\mathscr{A}}\left(r, \varepsilon^{1 / 2}\right)+E_{\mathscr{A}}\left(r, \varepsilon^{1 / 2}\right)\right) d r / 2 \varepsilon\right\} \exp \left\{i \omega_{\mathscr{A}}\left(t_{0}-\frac{b}{a} \varepsilon^{1 / 2}, \varepsilon^{1 / 2}\right)\right\} \\
& \cdot \exp \left\{-\frac{i c^{2}}{4 a} \log \left(\frac{c^{2}}{2 a}\right)\right\} e^{i c^{2} / 4 a} e^{-\pi c^{2} / 8 a} \frac{(1-i) c}{2 a^{1 / 2}} D_{\left(i c^{2} / 2 a\right)-1}((i-1) s) \\
= & \exp \left\{-i \int_{t_{0}-(b / a) \varepsilon^{1 / 2}}^{t(s, \varepsilon)}\left(E_{\mathscr{A}}\left(r, \varepsilon^{1 / 2}\right)+E_{\mathscr{A}}\left(r, \varepsilon^{1 / 2}\right)\right) d r / 2 \varepsilon\right\} \\
& \cdot \exp \left\{i \omega_{\mathscr{A}}\left(t_{0}-\frac{b}{a} \varepsilon^{1 / 2}, \varepsilon^{1 / 2}\right)\right\} \frac{(1-i) c}{2 a^{1 / 2}} \Xi_{1}(\tilde{t}, \varepsilon) .
\end{aligned}
$$

When $\tilde{t}=\varepsilon^{\kappa}$ with $\kappa \in\left(\gamma^{\prime}, \gamma\right)$,

$$
\begin{aligned}
\Xi_{1}(\tilde{t}, \varepsilon)= & \exp \left\{-\frac{i c^{2}}{4 a} \log \left(\frac{c^{2}}{2 a}\right)\right\} e^{i c^{2} / 4 a} e^{-\pi c^{2} / 8 a} D_{i c^{2} / 2 a-1}\left((i-1) \varepsilon^{\kappa-1 / 2}\right) \\
= & \exp \left\{-\frac{i c^{2}}{4 a} \log \left(\frac{c^{2}}{2 a}\right)\right\} e^{i c^{2} / 4 a} e^{-\pi c^{2} / 8 a} \frac{\sqrt{2 \pi}}{\Gamma\left(1-\frac{i c^{2}}{2 a}\right)} e^{-\pi c^{2} / 2 a} \\
& \cdot \exp \left\{-\frac{i a \varepsilon^{2 \kappa-1}}{2}-\frac{i c^{2}}{2 a} \log \left((i-1) a^{1 / 2} \varepsilon^{\kappa-1 / 2}\right)\right\}+O\left(\varepsilon^{p}\right) \\
= & \frac{\sqrt{2 \pi}}{\Gamma\left(1-\frac{i c^{2}}{2 a}\right)} e^{-\pi c^{2} / 4 a} \exp \left\{-\frac{i a \varepsilon^{2 \kappa-1}}{2}-\frac{i c^{2}}{4 a} \log \left(c^{2} \varepsilon^{2 \kappa-1}\right)+\frac{i c^{2}}{4 a}\right\}+O\left(\varepsilon^{p}\right) .
\end{aligned}
$$

The factors that multiply $\phi_{2}$ in (3.10) are

$$
\begin{aligned}
\exp & \left\{-i \int_{t_{0}-(b / a) \varepsilon^{1 / 2}}^{t(s, \varepsilon)}\left(E_{\mathscr{A}}\left(r, \varepsilon^{1 / 2}\right)+E_{\mathscr{B}}\left(r, \varepsilon^{1 / 2}\right)\right) d r / 2 \varepsilon\right\} \exp \left\{i \omega_{\mathscr{A}}\left(t_{0}-\frac{b}{a} \varepsilon^{1 / 2}, \varepsilon^{1 / 2}\right)\right\} \\
& \cdot \exp \left\{-\frac{i c^{2}}{4 a} \log \left(\frac{c^{2}}{2 a}\right)\right\} e^{i c^{2} / 4 a} e^{-\pi c^{2} / 8 a} D_{i c^{2} / 2 a}((i-1) s) \\
= & \exp \left\{-i \int_{t_{0}-(b / a) \varepsilon^{1 / 2}}^{t(s, \varepsilon)}\left(E_{\mathscr{A}}\left(r, \varepsilon^{1 / 2}\right)+E_{\mathscr{B}}\left(r, \varepsilon^{1 / 2}\right)\right) d r / 2 \varepsilon\right\} \\
& \cdot \exp \left\{i \omega_{\mathscr{A}}\left(t_{0}-\frac{b}{a} \varepsilon^{1 / 2}, \varepsilon^{1 / 2}\right)\right\} \Xi_{2}(\tilde{t}, \varepsilon) .
\end{aligned}
$$


When $\tilde{t}=\varepsilon^{\kappa}$ with $\kappa \in\left(\gamma^{\prime}, \gamma\right)$,

$$
\begin{aligned}
\Xi_{2}(\tilde{t}, \varepsilon)= & \exp \left\{-\frac{i c^{2}}{4 a} \log \left(\frac{c^{2}}{2 a}\right)\right\} e^{i c^{2} / 4 a} e^{-\pi c^{2} / 8 a} D_{i c^{2} / 2 a}\left((i-1) \varepsilon^{\kappa-1 / 2}\right) \\
= & \exp \left\{-\frac{i c^{2}}{4 a} \log \left(\frac{c^{2}}{2 a}\right)\right\} e^{i c^{2} / 4 a} e^{-\pi c^{2} / 8 a} \\
& \cdot \exp \left\{\frac{i a \varepsilon^{2 \kappa-1}}{4}\right\} \exp \left\{\frac{i c^{2}}{2 a} \log \left((i-1) a^{1 / 2} \varepsilon^{\kappa-1 / 2}\right)\right\}+O\left(\varepsilon^{p}\right) \\
= & \exp \left\{-\frac{i c^{2}}{4 a}\left(\log \left(\frac{c^{2} \varepsilon^{1-2 \kappa}}{4 a^{2}}\right)-\frac{3 \pi}{8 a}\right)\right\} \exp \left\{-\frac{\pi c^{2}}{8 a}+\frac{i c^{2}}{4 a}+\frac{i a \varepsilon^{2 \kappa-1}}{4}\right\}+O\left(\varepsilon^{p}\right) \\
= & \exp \left\{-\frac{i c^{2}}{4 a} \log \left(\frac{c^{2} \varepsilon^{1-2 \kappa}}{4 a^{2}}\right)+\frac{i c^{2}}{4 a}+\frac{i a \varepsilon^{2 \kappa-1}}{4}\right\}+O\left(\varepsilon^{p}\right) .
\end{aligned}
$$

Equations (3.14)-(3.17) describe the asymptotics of the inner solution in the positive time matching region. The asymptotics of the outer solution in this time interval contain two terms that correspond to the two terms in (3.13). The asymptotics of each term are evaluated by the techniques that led to (3.11) and (3.12) for the negative time outer solution. We consequently find that for $\tilde{t}=\varepsilon^{\kappa}$, the outer solution (3.13) equals

$$
\begin{aligned}
& \exp \left\{-i \int_{t_{0}-(b / c) \varepsilon^{1 / 2}}^{t(s, \varepsilon)}\left(E_{\mathscr{A}}\left(r, \varepsilon^{1 / 2}\right)+E_{\mathscr{B}}\left(r, \varepsilon^{1 / 2}\right)\right) d r / 2 \varepsilon\right\} \exp \left\{i \omega_{\mathscr{A}}\left(t_{0}-\frac{b}{a} \varepsilon^{1 / 2}, \varepsilon^{1 / 2}\right)\right\} \\
& \cdot\left(\Lambda_{\mathscr{A}} \exp \left\{-\frac{i a \varepsilon^{2 \kappa-1}}{2}-\frac{i c^{2}}{4 a}+\frac{i c^{2}}{2 a} \log \left(\frac{c \varepsilon^{(1 / 2)-\kappa}}{2 a}\right)\right\} \phi_{1}\left(t_{0}, \varepsilon^{1 / 2}\right)\right. \\
& \left.+\Lambda_{\mathscr{B}} \exp \left\{\frac{i a \varepsilon^{2 \kappa-1}}{2}+\frac{i c^{2}}{4 a}-\frac{i c^{2}}{2 a} \log \left(\frac{c \varepsilon^{(1 / 2)-\kappa}}{2 a}\right)\right\} \phi_{2}\left(t_{0}, \varepsilon^{1 / 2}\right)\right)+O\left(\varepsilon^{p}\right) .
\end{aligned}
$$

The formulas (1.6) and (1.7) for $\Lambda_{\mathscr{A}}$ and $\Lambda_{\mathscr{B}}$ were specifically chosen so that (3.18) would agree with the inner solution asymptotics described by (3.14)-(3.17), provided

$$
\omega(\varepsilon)=\omega_{\mathscr{A}}\left(t_{0}-\frac{b}{a} \varepsilon^{1 / 2}, \varepsilon^{1 / 2}\right)-\omega_{\mathscr{B}}\left(t_{0}-\frac{b}{a} \varepsilon^{1 / 2}, \varepsilon^{1 / 2}\right) .
$$

This completes the proof of Theorem 1.2.

Acknowledgements. It is pleasure to thank J. Avron, M. Klaus, and J. Segert for several useful discussions.

\section{References}

1. Avron, J. E., Seiler, R., Yaffe, L. G.: Adiabatic theorems and applications to the quantum Hall Effect. Commun. Math. Phys. 110, 33-49 (1987)

2. Berry, M. V.: Geometric amplitude factors in adiabatic quantum transitions, preprint

3. Berry, M. V.: Histories of adiabatic quantum transitions, preprint

4. Garrido, L. M.: Generalized adiabatic invariance. J. Math. Phys. 5, 335-362 (1964) 
5. Gradshteyn, I. S., Ryzhik, I. M.: Tables of integrals, series, and products. New York: Academic Press 1980

6. Hagedorn, G. A.: Adiabatic expansions near eigenvalue crossings. Ann. Phys. 196, 278-296 (1989)

7. Jakšić, V., Segert, J.: Exponential approach to the adiabatic limit and the Landau-Zener formula, preprint

8. Landau, L. D.: Collected Papers of L. D. Landau, Edited and with an introduction by D. ter Haar. New York: Gordon and Breach 1967

9. Lenard, A.: Adiabatic invariance to all orders. Ann. Phys. 6, 261-276 (1959)

10. Nenciu, G.: Adiabatic theorem and spectral concentration. Commun. Math. Phys. 82, 121-135 (1981)

11. Reed, M., Simon, B.: Methods of modern mathematical physics, Vol II. Fourier analysis, self-adjointness. New York: Academic Press 1975

12. Sancho, S. J.: $m^{\text {th }}$ order adiabatic invariance. Proc. Phil. Soc. London. 89, 1-5 (1966)

13. Simon, B.: Quantum mechanics for Hamiltonians defined as quadratic forms. Princeton, NJ: Princeton University Press 1971

14. Yajima, K.: Unpublished notes, March 1988

15. Yoshida, K.: Functional analysis. Berlin, Heidelberg, New York: Springer 1978

16. Zener, C.: Non-adiabatic crossing of energy levels. Proc. R. Soc. Lond. 137, 696-702 (1932)

Communicated by T. Spencer 
